\title{
Online Health Care Guidance using Artificial Intelligence
}

\author{
Prof. M.M. Waghmare ${ }^{1}$, Snehal Thopate ${ }^{2}$, Supriya Hume ${ }^{3}$, Shweta Dhole ${ }^{4}$, Komal Pansare ${ }^{5}$ \\ Professor, Computer Dept, SEC, Pune, India ${ }^{1}$ \\ Student, Computer Dept, SEC, Pune, India ${ }^{2,3,4,5}$
}

\begin{abstract}
Wellbeing Monitoring is more than once specified as one of the fundamental application regions for Artificial Intelligence. Versatile HealthCare is the reconciliation of portable registering and Health Monitoring. It is the utilization of versatile figuring innovations for enhancing correspondence among patients, doctors, attendants, assistant and other medicinal services specialists. As cell phones have turned into an inspirable piece of our life can incorporate medicinal services more flawlessly to our regular day to day existence. It empowers the conveyance of exact restorative data at whatever time anyplace by method for cell phones. The utilization of Electronic Medical Record (EMR) frameworks can resolve some of the long standing therapeutic wasteful aspects regular in creating nations. This paper exhibits the plan and usage of a proposed outpatient administration framework that empowers effective administration of a patient medicinal detail. We exhibit a framework to make meetings with medicinal professionals by incorporating a proposed Android based versatile application with a chosen open source EMR framework. The possibility of this venture is to give an association with a setup that will be useful to that particular association as well as the patient connected with the association also. The patient will have an application particularly intended for them which will push them to contact with a specialist, collaborate with him.
\end{abstract}

Keywords: Electronic Medical Record, Mobile HealthCare, Mobile Communication.

\section{INTRODUCTION}

The goal of the venture is to give fundamental medicinal administrations online to clients independent of their area. Clients can associate through their home web or approach any adjacent booth to get these administrations. The inspiration to fabricate the framework is that not very many or no specialists are accessible at remote areas, restricted hour administrations and absence of advanced medicinal supplies and no patients history/lab information administration

\section{EXISTING SYSTEM}

In present frameworks there is not really any therapeutic administration accessible in remote areas. People requiring restorative administrations frequently need to travel long separations. Indeed, even in urban ranges the administration is in some cases not accessible instantly. Patients and specialists are barely to speak with every others.

Furthermore patients needed to sit tight for long time keeping in mind the end goal to convey to the specialist.

\section{PROPOSED SYSTEM}

The proposed framework is an online application which is accessible constantly. The framework gives subtle elements of medicinal administrations on the web and permits clients to interface with specialists and other therapeutic faculty. The patients and specialists are cooperating with talking applications at their relative spots. At first the specialists had got the patients points of interest in the online by help of administrator individuals keeping in mind the end goal to impart in visit.

\section{LITERATURE SURVEY}

1. Amiya Tripathy, Ajit Varghese, Nikita Chabhaiya and Bejoy Anthony, MediAssistEdge Simplifying finding strategy enhancing quiet specialist network, Don Bosco Institute of Technology, Under Graduate venture report 2014

The belief system of remote analysis in genuine sense is made conceivable with the assistance of the two helping equipment gadgets which comes coordinated with the framework. The second objective of conclusion of illness is actualized by the DocBot subsystem which is an Artificial Intelligence (AI) motor to associate with patients. It has an interface wherein a representation of a specialist will be available with an answer box. The client connects with the manmade brainpower motor like typical talking. In light of the contributions from the client the AI motor will give conclusion. The specialists will likewise have the capacity to educate the AI motor. The patients that require second level conclusion will be alluded to the healing center.

2 J. Allen, Photoplethysmography and its application in clinical physiological estimation, Physiological Measurement, Vol. 28, pp. R1R39, 2007. 
Photoplethysmography (PPG) is a noninvasive optical strategy for distinguishing microvascular blood volume changes in tissues. Its usability, minimal effort and comfort make it an alluring region of research in the biomedical and clinical groups. The presentation of quick computerized cameras into clinical imaging checking and analysis frameworks, the yearning to diminish the physical limitations, and the conceivable new bits of knowledge that may originate from perfusion imaging and mapping motivated the development of the ordinary PPG innovation to imaging PPG (IPPG). IPPG is a noncontact strategy that can distinguish heart-produced beat waves by method for fringe blood perfusion estimations. Since its commencement, IPPG has pulled in noteworthy open intrigue and gave chances to enhance individual medicinal services.

3. DanilLakens, Using a Smartphone to Measure Heart Rate Changes amid Reviewed Happiness and Anger, IEEE exchanges on full of feeling figuring, Vol. 4, Issue. 2, April-June 2013.

This study exhibits the practicality of measuring heart rate (HR) contrasts connected with passionate states, for example, outrage and bliss with a cell phone. Tenderfoot experimenters measured higher HRs amid remembered outrage and joy (recreating discoveries in the writing) outside a research center environment with a cell phone application that depended on photoplethysmography.

4. "A customer server design for circulated estimation systems",IEEE Exchanges on(Volume:47,Issue:5) Oct 1998.

This paper portrays a customer server design for the remote control of instrumentation over the Internet organize. The proposed arrangement permits multiuser, multi-instrument sessions by method for a queueing and instrument locking capacity. Customer applications can be effortlessly created by utilizing traditional abnormal state programming dialects or all around surveyed virtual instrumentation systems. Execution tests are accounted for; they demonstrate the low overhead because of system operation concerning the immediate control of instrumentation.

5. "Portable based human services administration utilizing articial knowledge", Technologies for Sustainable Development, 2015 International Conference on Feb 2015 In this developing time of innovation it is important to have an appropriate social insurance administration framework which ought to be penny percent exact additionally ought to be versatile so that each individual convey with it as customized human services framework. The medicinal services administration framework which will comprise of portable based Heart Rate Measurement so that the information can be exchanged and conclusion in light of heart rate can be given rapidly a tick of catch. The framework will comprise of video conferencing to associate remotely with the Doctor. The Doc-Bot which was produced before is currently being exchanged to portable stage and will be further exceptional for finding of regular ailments. The framework will likewise comprise of Online Blood Bank which will give up and coming insights about accessibility of blood in various healing centers.

\section{ARCHITECTURE}

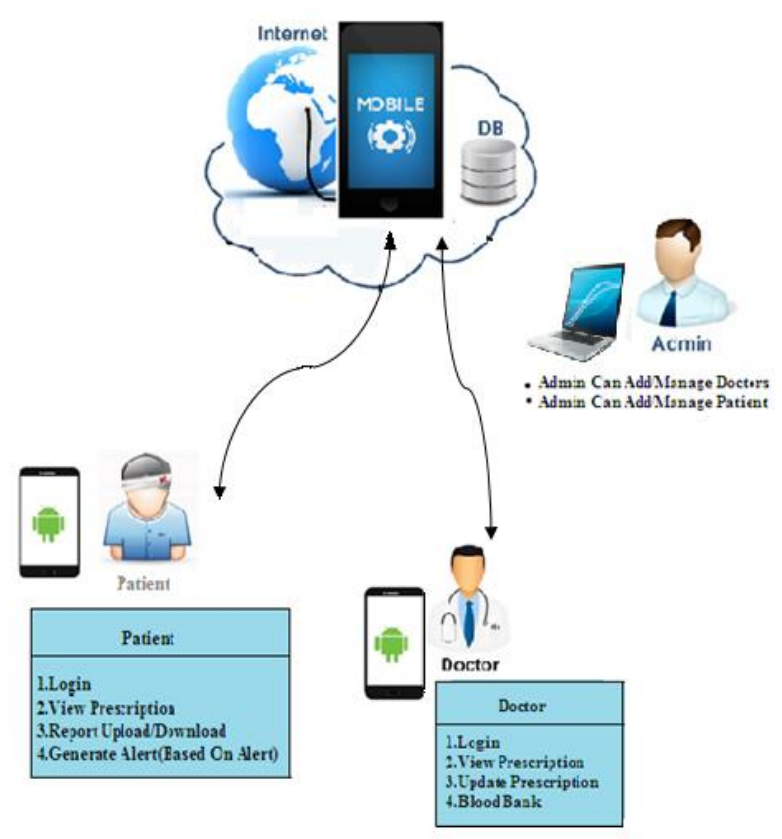

The Health Management System includes two fundamental areas, the main segment is the UI which is implied for data show and client collaboration with the framework while the second segment (master framework) involves the induction motor and the knowledge base where the demonstrative focus is arranged. The handling mode is exceedingly intuitive also, the preparing calculation which was utilized to accomplish the required assignment is plot beneath:

1. At the point when the framework is begun, the Welcome Screen is shown with a catch named "ENTER". This permits the patient to see the Login window.

2. The Login window will be shown for the patient to login to the framework.

3. The client can either tap on the" new client interface name" if that is the patient's first time of utilizing the product or enter his/her username and secret word to see the following windows

4. In the event that the patient has tapped the connection for the new client, the persistent requirements to enter his/her subtle elements with the goal that it can then be put away into the database. In the event that the patient has entered the username and secret word, the patient's username and secret word can either be unapproved. In the event that the username and secret word of the patient has been approved, there is a frame that would be shown in which the client can either click the "restorative" or the "offer assistance" menu strip bar. In the event that 
Vol. 5, Issue 12, December 2016

"therapeutic" was picked then the patients can then have admittance to the finding page to choose and deselect his/her side effects, get the pharmaceutical and wellbeing tips for his/her sickness. In the event that the patient's username and watchword of the patient have not been approved this implies the username or watchword is invalid, then the understanding needs to tap on the new client connect mark to enlist another username and secret word.

5. The patient can likewise check his/her Body Mass Record (BMI) to check on the off chance that he/she is stout, ordinary or overweight. This tags along with helpful wellbeing tips that can help the patient conform from either being hefty overweight to an ordinary Body Mass Index.

6. The patient can likewise get his/her points of interest in the wake of having been analyzed by the wellbeing administration framework.

7. At the point when the client wraps up the framework, the client leaves the framework by shutting the windows.

\section{CONCLUSION}

Medicinal services administration framework is created with the end goal of wager torments for individuals wellbeing. The portability is a vital variable of this application. It gives simpler technique to the client to speak with the specialist and show signs of improvement and speedier consultancy. Our framework if actualized on an authoritative level can be extremely effective to give wellbeing administration offices to the client of this administration framework. The framework can give minimal effort and quality wellbeing administrations to the patients and keep up certain wellbeing standard by regular ailment conclusion too.

\section{REFERENCES}

[1] Amiya Tripathy ,Ajit Varghese, Nikita Chabhaiya and Bejoy Anthony, MediAssistEdge Simplifying diagnosis procedure improving patient doctor connectivity, Don Bosco Institute of Technology, Under Graduate project report 2014

[2] Bing Nan Li, Ming-Chui Dong, Sam Chao, On decision making support in blood bank information systems. Expert Syst. Appl. Vol. 34, Issue 2, pp.1522-1532, 2008

[3] Priyanka V. Mhamunkar, Krishna S. Bansode and Laxman S. Naik, Android Application to get Word Meaning through Voice,International Journal of Advanced Research in Computer Engineering and Technology, Vol. 2, Issue 2, February 2013.

[4] J. Allen, Photoplethysmography and its application in clinical physiological measurement, Physiological Measurement, Vol. 28, pp. R1R39, 2007.

[5] DanilLakens, Using a Smartphone to Measure Heart Rate Changes during Recalled Happiness and Anger, IEEE transactions on affective computing, Vol. 4, Issue. 2, April-June 2013.

[6] Healthcare IT News, Electronic Medical Record (EMR), Internet: www.healthcareitnews.com/directory/record-emr, 2014 [June 18, 2014].

[7] George. Gousios , "A Comparison of portable Dynamic web content Technologies for the apache server", 3rd International Administration and Networking Conference Proceedings.

[8] Web Database Connectivity Methods (using Mysql) in Windows Plat- form, Information and Communication Technologies, 2006. ICTTA '06. 2nd (Volume:2 ).
[9] "A client-server architecture for distributed measurement systems",IEEE Transactions on(Volume:47,Issue:5) Oct 1998.

[10] "Mobile based healthcare management using articial intelligence", Technologies for Sustainable Development, 2015 International Conference on Feb 2015 